\title{
Introduction the paper: Posterior fossa decompression and the cerebellum in Chiari type II malformation: A preliminary MRI study, by Salman M
}

\author{
Gianpiero Tamburini
}

Received: 6 December 2010 / Accepted: 6 December 2010/Published online: 25 January 2011

(C) Springer-Verlag 2011

\section{Introduction}

The present paper focuses on the possible role of posterior fossa decompression performed in early infancy as a potential aid in preventing cerebellar atrophy progression in children with spina bifida aperta and Chiari II malformation. MR findings in a very limited sample group of children (four cases), operated on in the first 2 years of life for their Chiari II malformation, are compared with those obtained in a group of 28 Chiari II malformation not operated on patients and those of 10 age-matched normal control subjects. An increase in mean MR CSF volume within the cerebellar fissures and the fourth ventricle in operated children, if compared with the CSF volume in non-operated patients and the one of normal subjects is the base for indicating a very early surgical correction. In other words, the operated children would have more space available for postnatal cerebellar growth.

There are a number of controversial issues concerning this proposal, that have suggested the claim for comments from people experienced in the field; only to cite some of the possible discussion points:
1) The hindbrain embryological development is completed by the second month of embryological life, and hindbrain abnormalities are already completely defined by that time.

2) The rate of children with open spina bifida becoming symptomatic for a Chiari II malformation is relatively low and might not be enough to justify an early preventive surgery, even in a selected proportion of patients.

3) As the same authors state, in spite of a lower rate of Chiari II malformations in patients with open spina bifida operated on in utero, previous studies suggest that cerebellar structures width on postnatal MR is not different, when the Chiari II malformation is present, if the repair of the spinal defect is performed during fetal life or after birth.

4) The surgical risks of a preventive posterior cranial fossa decompression in neonates should not be underestimated and balanced with the only eventual advantage I can foresee of this kind of surgical timing, which is the possible prevention of hindbrain signs in case of shunt malfunction in shunted patients.

The invited comments are published thereafter.

G. Tamburini $(\bowtie)$

Catholic University Medical School,

Rome, Italy

e-mail: gianpiero.tamburrini@rm.unicatt.it 